\title{
VALIDACIÓN DEL DISPOSITIVO IMPERIAL COLLEGE SURGICAL ASSESMENT DEVICE (ICSAD) PARA BLOQUEO DE NERVIO CIATICO POPLÍTEO EN UN MODELO SIMULADO
}

Fuentes Amaya Carlos ${ }^{1}$, Araneda Vilches Andrea ${ }^{1}$, Miranda Hiriart Pablo ${ }^{1}$, Carmona Barra Javiera ${ }^{1}$, Altermatt Couratier Fernando ${ }^{1}$, Corvetto Aqueveque, Marcia ${ }^{1}$

1 División de Anestesiología, Escuela de Medicina, Pontificia Universidad Católica de Chile.

Introducción: El dispostivo Imperial College Surgical Asessment Device (ICSAD) ha mostrado validez para discriminar competencias técnicas en diferentes procedimientos de anestesia regional ${ }^{1,2}$; sin embargo, aún no se conoce su verdadera utilidad dentro del proceso de entrenamiento para bloqueos de nervio ciatico poplíteo. Objetivo General: Determinar la validez de constructo del dispostivo ICSAD como herramienta para evaluar competencias técnicas entre operadores de distinta experticia durante un bloqueo ciático poplíteo simulado.

Material y Métodos: Posterior a la aprobación del comité de ética de la Pontifica Universidad Católica de Chile, 20 operadores con diferentes niveles de experticia fueron reclutados y categorizados en 2 grupos: novatos y expertos. Cada uno de ellos realizó un bloqueo ciatico poplíteo bajo visión ecográfica en plano, sobre un fantoma especialmente diseñado para este propósito. Los sensores del dispositivo ICSAD fueron ad- heridos al dorso de las manos de cada operador registrando las variables: distancia total recorrida por ambas manos (DTR), distancia total recorrida por cada mano (escaneo y aguja), número de movimientos de ambas manos (NMM) y tiempo total del procedimiento (TTP). Para el análisis estadísico, se dividió el bloqueo en dos fases: (1) escaneo y (2) punción. Las mediciones fueron ajustadas por mano dominante y las diferencias entre ambos grupos fueron establecidas mediante el test de Mann Withney considerando significativo un valor $\mathrm{p}<\mathrm{a} 0,05$.

Resultados: Se midió a un total de 20 sujetos: 10 residentes de primer año en el grupo novatos y 10 staff de anestesia en el grupo expertos. Durante la fase 1 sólo el TTP fue significativamente menor en el grupo de expertos $(p=0,025)$; para la fase 2, las variables DTR de ambas manos, DTR de mano de escaneo (probe) y TTP mostraron diferencias estadísticamente significativas entre los grupos (tabla 1). Al ajustar por mano

Tabla 1

\begin{tabular}{|lccc|}
\hline & $\begin{array}{c}\text { Novatos } \\
(\mathbf{n = 1 0})\end{array}$ & $\begin{array}{c}\text { Expertos } \\
(\mathbf{n = 1 0 )}\end{array}$ & valor p \\
Distancia mano escaneo (m) & 0,95 & 0,28 & $<0,001$ \\
Distancia mano punción (m) & $(0,59-8,08)$ & $(0,17-0,3)$ & 0,041 \\
& 3,56 & 2,03 & $(1,27-2,43)$ \\
Distancia total (m) & $(1,66-4,03)$ & 2,3 & 0,003 \\
Número de movimientos & 4,8 & $(1,4-2,7)$ & 0,739 \\
Tiempo de Procedimiento (s) & $(3,4-6,1)$ & 7 & $<0,001$ \\
\hline
\end{tabular}

Valores expresados en mediana y rango intercuartil; Distancias expresadas en metros (m) y tiempos en segundos (s);

Valores P obtenidos al comparar grupos con el test de Mann Whitney. 
dominante, la DTR de la mano de escaneo fue la única capaz de discriminar competencias entre operadores $(p<0,001)$. Finalmente al obtener los valores sumativos de ambas fases para DTR de mano de escaneo (probe), DTR de ambas manos y TTP, se mantuvieron las diferencias entre novatos y expertos de forma significativa con valores $\mathrm{p}$ de $0,009,0,018 \mathrm{y}<0,001$ respectivamente. Conclusiones: Este es el primer estudio que utiliza el dispositivo ICSAD como evaluador de habilidades para un bloqueo ciático poplíteo; provee información cuantitativa complementaria a las escalas globales validadas y confirma que la DTR de la mano de escaneo discrimina entre operadores. Se requieren otros estudios para de- terminar la efectividad clínica de un programa de entrenamiento en simulación que use esta herramienta.

\section{Referencias}

1. Hayter MA, et al. Validation of the Imperial College Surgical Assessment Device (ICSAD) for labour epidural placement. Can J Anaesth 2009; 56: 419-26.

2. Chin KJ, et al. Hand motion analysis using the imperial college surgical assessment device: validation of a novel and objective performance measure in ultrasound-guided peripheral nerve blockade. Reg Anesth Pain Med 2011; 36: 213-9. 